\title{
Apply Forward Simulations in 2D ERT Monitoring Program of a New Embankment Dam
}

\author{
PolinWu, Chunhung Lin, Hsinchang Liu, \\ Assistant Researcher, Natural Hazard Prevention and Water \\ Environment Research Center, \\ National Chiao Tung University \\ Hsinchu, Taiwan.
}

\begin{abstract}
Resistivity method is generally considered suitable for investigation of seepage problems in earthen embankments. Construction of a large embankment dam in Taiwan was recently completed, providing a good opportunity for ERT monitoring of the entire water filling process and future performance. The proposed monitoring program consists of longitudinal ERT survey lines on the crest and downstream shell. One transverse survey line was added to study the seepage behavior during water filling. 2D ERT investigation on many geo-engineering structures, such as dikes or dams, violates the $2 \mathrm{D}$ assumption due to the $3 D$ topology and internal zonation, leading to potential artifacts in the inverted $2 \mathrm{D}$ resistivity section. The monitoring survey lines are primarily in the longitudinal direction. The resistivity of neighboring zone of different material and the topology change on the two sides of the survey line may cause 3D effects on the 2D inverted resistivity section right beneath the line. This presentation introducesforwardmodel simulationsfor planning of the survey and evaluating potential problems of 2D ERT investigation on embankments.
\end{abstract}

Keywords-Forward Simulations; $2 D \quad$ ERT; New Embankment Dam;Resistivity method

\section{INTRODUCTION}

Current practice of dam safety assessment ismainly based on visual inspection and sparse geotechnical monitoring data. Visual inspections do not provide information inside the dam, while monitoring instrument provides engineering parameters only at discrete points with limited spatial coverage of the dam. There is a growing demand for the use of non-intrusive geophysical techniques to see into the dam and facilitate early detection or diagnosis of anomalous phenomena $^{[6]}$. In particular, time-lapse geophysical measurements are appealing for dam safety assessment. Construction of a large embankment dam for the Hu-Shan Reservoir in Taiwan was recently completed, providing a good opportunity for geophysical monitoring of the entire water filling process and future performance. A geophysical monitoring program was devised for the new dam that includes electrical resistivity tomography (ERT), self potential (SP), and multichannel analysis of surface wave (MASW), as shown in Fig. 1. The dominant potential failure mode of an earth dam is seepage-related problems, justifying the use of ERT and SP. MASW was used to measure the dynamic property (i.e. shear-wave velocity) for the analysis of dynamic response and evaluate the strength condition of

\author{
ChihpingLin* \\ Professor of Civil Engineering, \\ National Chiao Tung University \\ Hsinchu, Taiwan.
}

the stabilizing downstream shell. This presentation focuses on the discussion of ERT.

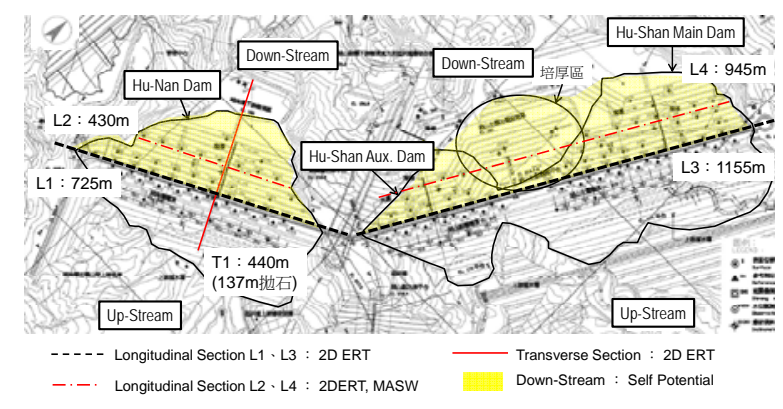

Fig.1The geophysical monitoring program in the Hu-Shan Reservoir

The electrical resistivity tomography (ERT) is one of the most widely used geophysical methods in geological, hydrogeological, and geo-environmental investigationsp ${ }^{[3,4]}$. Although 3D ERT is now available, 2D ERT remains to be state-of-the-practice due to its simplicity in field works and less requirement in space and equipment.Since the purpose of the geophysical monitoring is to scan potential anomalies in any location of the dam, the whole extent of the dam in the longitudinal direction are covered by 2D ERT survey lines both on the core and downstream shell. Only one transverse survey line is planned to provide cross-sectional information on seepage behavior during water filling. 2D ERT assumes the ground condition perpendicular to the survey line is homogeneous. This assumption is apparently violated when conducting 2D ERT surveys in the longitudinal direction of the dam due to variation of topology and filled material in the transversedirection ${ }^{[5]}$. The ability of 2D ERT to detect seepage anomalies under the influence of 3D effects needs to be investigated. Dams are complex 3D structures. Even the transverse survey line does not conform to the 2D condition due to abrupt elevation change of the valley. This presentation introduces 3D forward model simulationsfor planning of the survey and evaluating potential problems of 2D ERT investigation on embankments.

\section{Modeling SETUP}

The 3D modeling of resistivity survey was conducted using the finite element software COMSOL Multiphysics ${ }^{\circledR}$ (COMSOL Group, 2011). The COMSOL Multiphysics ${ }^{\circledR}$ AC/DC module can simulate the electrical current and 
potential field of the resistivity survey. The simulated data was inverted for resistivity section using RES2DINV ${ }^{[2]}$ as in the field investigation.

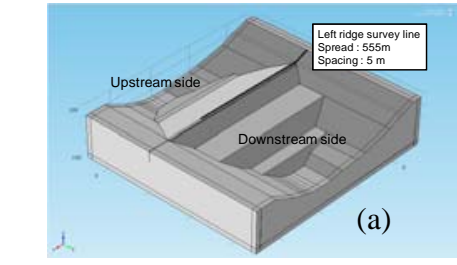

(b)
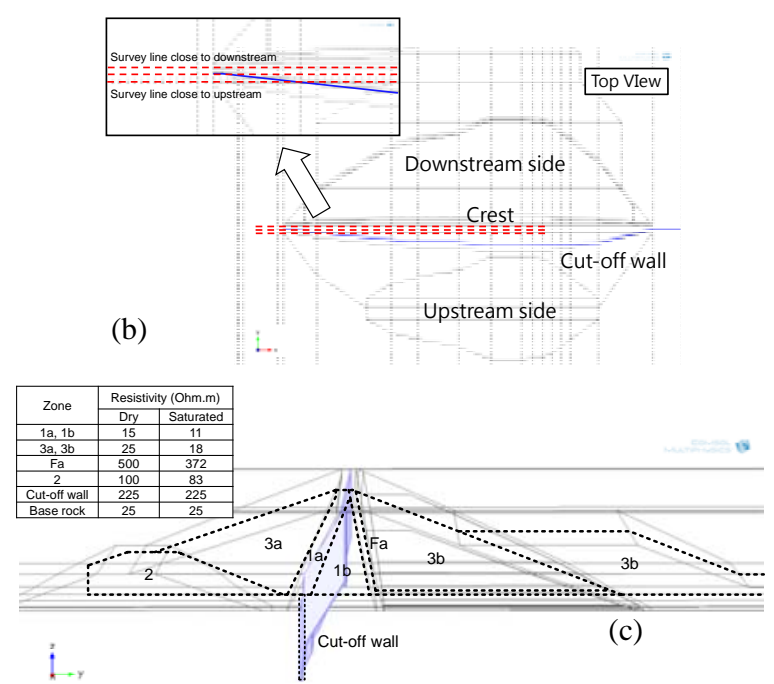

Fig.23D modeling of the Hu-Shan dam: (a) overhead view; (b) top view; (c) transparent side view showing resistivity parameters of different zones

The Hu-Shan reservoir actually consists of three embankment dams separated by ridges. The entire dam is divided into Hu-Shan Main Dam, Hu-Shan Auxiliary Dam, and Hu-Nan Dam, as depicted in Fig. 1. The forward simulation model of this study is based on the geometry and design parameters of the Hu-Nan Dam. The modeling geometry and resistivity parameters of different partition (including core, shell, filter, foundation cut-off wall, and rock foundation) in dry (before water filling) and saturated condition (after water filling) are shown in Fig. 2.

Both the transverse survey line (T1) and the longitudinal survey lines were modeled. In the transverse survey line, the investigation coverage and alternative survey method after water filling are discussed. For the longitudinal survey line, the internal 3D effect due to zonation of compacted soil and external 3D effect due to change of reservoir water level are examined. Preliminary field surveys suggest the use of Wenner-Slumberger electrode array. So the same configuration is used for numerical simulations.

\section{RESULTS AND DISCUSSION}

\section{A. Performance of survey line configuration}

The upstream shell is covered by a riprap layer to protect the dam from erosion. The existence of the riprap makes it rather difficult to insert electrode with good contact. Three possible configurations were simulated: (a) land electrodes covering both upstream and downstream sides; (b) land electrodes covering only the downstream side; (c) combination of floating and land electrodes. The results are shown in Fig. 3. Without electrodes covering the upstream shell, the ERT image cannot sufficiently cover the core. Electrodes on the upstream side are necessary and underwater electrodes may be used for the submerged part for the sake of long-term performance. In case of failure of underwater electrodes, an alternative survey using floating electrodes was also simulated. As shown in Fig. 3(c), the result of utilizing floating electrodes on the upstream side is comparable to that of using underwater electrodes.

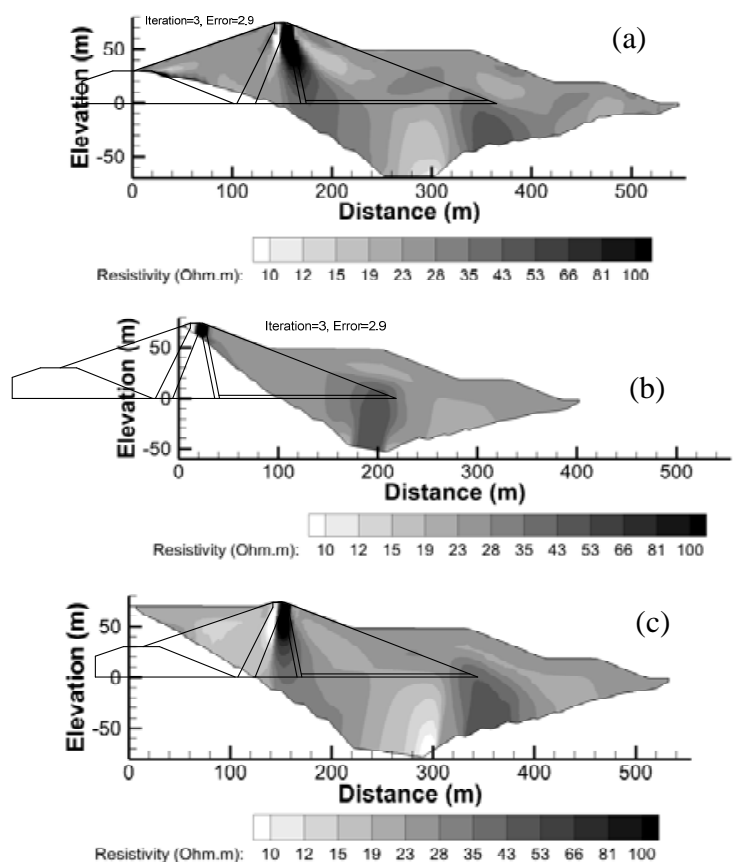

Fig. 3Simulated ERT results for (a) land electrodes with full coverage; (b) electodes covering only the downstream side; (c) configuration with floating electrodes

\section{B. Evaluation of $3 D$ effects}

For the longitudinal survey lines, 3D effects may arise from the interior material variation as well as changes in topography andexternalwater level changes in the transverse direction.

\section{1)Internal 3D Effect}

The longitudinal survey lines on the dam crest are mainly used to inspect the condition of the core. Although any location on the dam crest is on top of the core, surveying at different locations may result in different degree of 3D effects the influence of filter, shell, and foundation cut-off wall depending on the survey location. This was studied by numerical simulations during the planning of the geophysical monitoring program. 
As shown in Fig. 2(b), survey lines at the center, at the upstream edge, and at the downstream edge of the dam crest were simulated. The spread length of each survey sub line is $555 \mathrm{~m}$ with $5-\mathrm{m}$ electrode spacing. The time-lapse analysis scenario of leakage is shown in Fig. 4, and the results of L11 (first $555 \mathrm{~m}$ of the entire line) before water filling are shown in Fig. 5. All three lines show signs of anomalies after time-lapse difference. This is more apparent for the lines located at the upstream edge and crest center. The reason may be explained by the fact that the filter,the resistivity of the filter is much higher than the core and shell to affect the anomalies detection. These simulations are underway to examine the sensitivity of different survey location to the seepage anomaly to finalize the layout for long-term monitoring.

\section{2)External 3D Effect}

After water filling, the variation of water level may alter the 3D effect on 2D ERT results. This is demonstrated by a simulation scenario where the water level drops by $5 \mathrm{~m}$ from the maximum level (EL. 211.5m) to EL. 206.5m. The timelapse analysis scenario was shown in Fig. 6, and the results are shown in Fig. 7. In Fig. 6, the resistivity of the dam body is kept constant during rapid drawdown (in Time2), and the internal water level is drop with time (in Time3). As shown in Fig. 7(a), the leakage area was easy to detection because of time-lapse difference with same reservoir level condition. In Fig. 7(b), it was not easy to detect anomalies because the time-lapse difference indicates significant false resistivity change due to change in reservoir water level. Further research should be conducted to remove external 3D effect, so that the 2D ERT can be used to more reliably detect interior anomalies. Alternatively, the ERT survey may be automated to acquire measurement data of higher monitoring frequency and time-lapse differential analysis is performed on results at different times but the same reservoir water level.

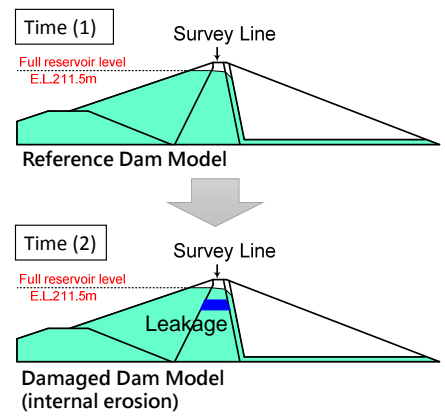

Fig. 4Time-lapse analysis of leakage scenario with the same reservoir level

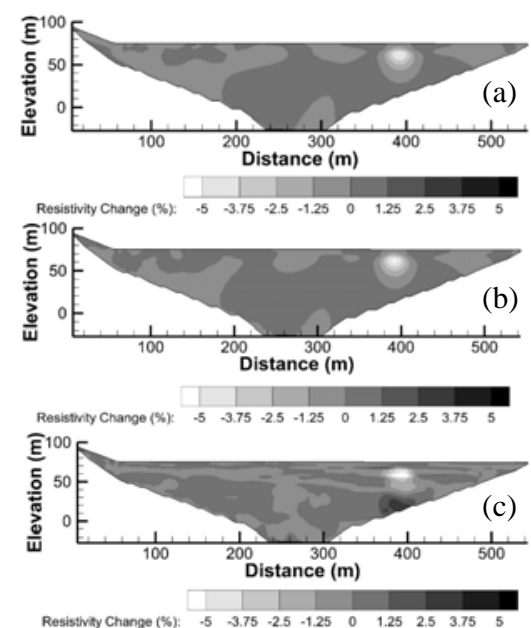

Fig.5Simulated ERT results for the survey line (a) at the upsteam edge, (b) cenerline of crest, and (c) downstream edge

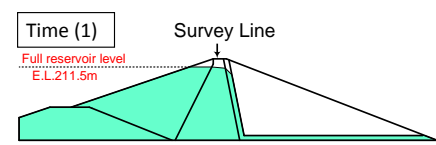

Short term water level change

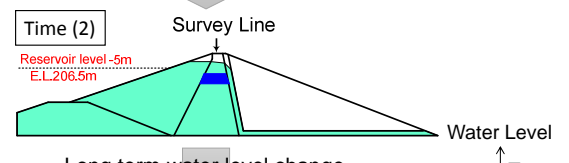

Long term water level change
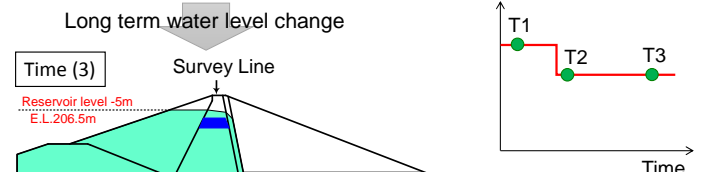

Fig.6Time-lapse analysis of leakage scenario for the different reservoir level

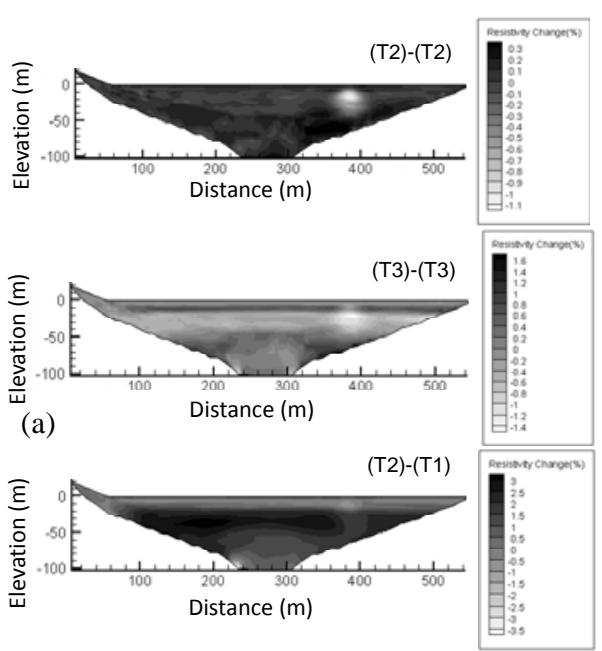




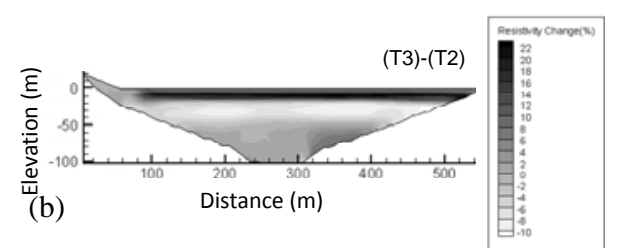

Fig.7Time-lapse analysis of simulated ERT results (a) same reservoir level and (b) different reservoir level

TABLE I. ANOMALIES DETECTION FROM SIMULATED RESULT

\begin{tabular}{|c|c|c|c|c|}
\hline \multicolumn{5}{|c|}{ Same reservoir level } \\
\hline \multirow{17}{*}{$\begin{array}{c}\text { scale } \\
\left(m^{\wedge} 2\right)\end{array}$} & Depth & $10 \mathrm{~m}$ & $20 \mathrm{~m}$ & $30 \mathrm{~m}$ \\
\hline & \multicolumn{4}{|c|}{ Fully empty } \\
\hline & $5 * 5$ & $\checkmark$ & $\checkmark$ & $\checkmark$ \\
\hline & $3 * 3$ & $\checkmark$ & $\checkmark$ & $\checkmark$ \\
\hline & $1 * 1$ & $\checkmark$ & $\checkmark$ & - \\
\hline & \multicolumn{4}{|c|}{ Initial empty } \\
\hline & $5 * 5$ & $\checkmark$ & $\checkmark$ & $\checkmark$ \\
\hline & $3 * 3$ & $\checkmark$ & $\checkmark$ & $\checkmark$ \\
\hline & $1 * 1$ & $x$ & - & - \\
\hline & \multicolumn{4}{|c|}{ Fully leakage } \\
\hline & $5 * 5$ & $\checkmark$ & $\checkmark$ & $\checkmark$ \\
\hline & $3 * 3$ & $\checkmark$ & $\checkmark$ & - \\
\hline & $1 * 1$ & $x$ & - & - \\
\hline & \multicolumn{4}{|c|}{ Initial leakage } \\
\hline & $5 * 5$ & $\checkmark$ & $\checkmark$ & $\checkmark$ \\
\hline & $3 * 3$ & $\checkmark$ & - & - \\
\hline & $1 * 1$ & - & - & - \\
\hline
\end{tabular}

\section{CONCLUSIONS}

Construction of a large embankment dam in Taiwan was recently completed, providing a good opportunity for ERT monitoring of the entire water filling process and future performance.Dams are complex 3D structures. Even the transverse survey line may not conform to 2D condition due to abrupt elevation change of the valley. 2D ERT assumes the ground condition perpendicular to the survey line is homogeneous. This assumption is apparently violated when conducting 2D ERT surveys in the longitudinal direction of the dam due to variation of topology and filled material in the transverse direction.

This study utilized 3D modeling to examine 3D effects on 2D ERT and investigate the optimal survey location and configuration. The transverse survey line is challenging due to the riprap on the upstream shell. To have sufficient imaging coverage on the core, it is planned to use underwater electrodes for the submerged part during water filling. The 3D effect is evident for the longitudinal survey lines and the degree of influence depends on the exact location of the survey line. More importantly, the contribution of 3D effect from changes in reservoir water level may interfere with the detection of seepage anomalies. Further research should be conducted to remove external 3D effect. Alternatively, the
ERT survey may be automated to acquire measurement data of higher monitoring frequency and time-lapse differential analysis is performed on results at different times but the same reservoir water level.

\section{ACKNOWLEDGEMENTS}

The authors are grateful for thesponsorship from the Water Resources Planning Institute, Water Resources Agency, Taiwan.

\section{REFERENCE}

[1] COMSOL Group, 2011, COMSOL Multiphysics Finite Element Analysis Software Ver. 4.2a, Official Site: http://www.comsol.com/.

[2] Geotomo Software, 2011, RES2DINV Ver. 3.59, Rapid 2-D Resistivity and IP inversion using the least-squares method, available at www.geoelectrical.com.

[3] Lin, C. P., Hung, Y. C., Wu, P. L., and Yu, Z. H., 2014, Application of ERT to Investigation of Abnormal Seepage: A Case Study at the Hsin-Shan Earth Dam in Taiwan: Journal of Environmental and Engineering Geophysics, 19(2), 101112.

[4] Wu, P. L., Lin, C. P., Hung, Y. C., 2012a, Evaluation of 2D ERT for Investigating Abnormal Seepages at an Earth Dam: 2012 KSEG International Symposium on Geophysics for Discovery and Exploration.

[5] Wu, P. L., Lin, C. P., Hung, Y. C., 2012b, 3D Effects on 2D Electrical Resistivity Tomography - A Case study in Investigation of Hsin-Chu Fault: 2012 KSEG International Symposium on Geophysics for Discovery and Exploration.

[6] Voronkov, O.K., Kagan, A.A., Krivonogova, N.F., Glagovsky, V.B., Prokopovich, V.S., 2004, Geophysical methods and identification of embankment dam parameters: in Proceedings of the 2nd International Conference on Geotechnical and Geophysical Site Characterization, 593599. 